\title{
Patrón autoorganizativo comunicativo de la empresa: de la intención a la acción
}

RICARDO ARIZA LÓPEZ

“Miguel” significa “QQuién es Dios?”. Es el único arcángel cuyo nombre es una pregunta. A esa pregunta el Enemigo no sabe responder. Ésa es la espada del arcángel.

\section{RESUMEN}

De acuerdo con lo establecido en la intencionalidad investigativa del proyecto Marketing holístico, la empresa está enmarcada dentro de los supuestos que implican considerarla como un sistema vivo constituido por: un patrón de organización (la configuración de las relaciones que determinan las características esenciales del sistema); una estructura (la corporización física del patrón de organización del sistema); y el proceso vital (la actividad involucrada en la continua corporización física del patrón de organización del sistema).

Este artículo propone una lectura sistémica de carácter complejo para determinar el patrón autoorganizativo comunicativo de la empresa (sea cual fuere ella y el sector económico y productivo donde se encuentre calificado o inscrito) que posibilite, en el transcurso de la investigación marketing holístico, considerar las bases para la construcción de modelos de sistemas viables para plataformas estratégicas competitivas.

\section{PALABRAS CLAVE}

Comunicación, patrón autoorganizativo, empresa, holístico, sistémico, conversación, aprendizaje.

\section{Patrón de autoorganización comunicativa}

La evaluación del patrón de autoorganización comunicativa de la empresa nos permitirá determinar si esta ha tenido una reorganización continua, dinámica y pensada de manera integral y sistémica, condición que viene a ser indispensable para su adaptación a entornos cambiantes de mercado.

Desde el punto de vista del sistema, diríamos que este reajusta su estructura interna para seguir siendo compatible con su entorno. El sistema se adapta. Es la piel de gallina frente al frío, la agricultura frente a la escasez o frente al riesgo de la caza, los semáforos frente a un aumento de tráfico. Cuando ello no es posible por una perturbación (fluctuación) en uno de los términos que no puede ser absorbida por una respuesta de los otros tres, la adaptación se rompe y el sistema entra en crisis. El sistema entonces, o bien se extingue, o bien cambia bruscamente a otra (nueva, imprevisible) estructura, se autoorganiza en clara rebelión contra su entorno. A partir de esta visión compleja de la empresa, diríamos que una de las condiciones indispensables para su desarrollo y adaptación a nuevos mercados sería la autopoiésis. Esto quiere decir que la empresa, para lograr su realización efectiva (el cumplimiento de su finalidad y objetivos), debe generar su propia organización acorde con la propuesta global de sentido de empresa como sistema integral, holístico y complejo.

Para analizar, comprender y evaluar la situación actual de la empresa consideramos indispensable conocer su 
historia. La búsqueda de este conocimiento histórico nos aportará la información necesaria para determinar con mayor validez las razones que explican bajo qué condiciones estructurales y en qué circunstancias de entorno la empresa ha construido y posesionado una razón de ser. Generalmente las circunstancias que rodean a los diferentes momentos históricos de la vida de la empresa hacen surgir ciertos valores e ideologías que afectan el funcionamiento mucho más allá que los planes operativos y los programas estratégicos o estructurales elaborados para superar las contingencias propias de una administración empresarial. Pensar y crear un sentido de identidad y posicionamiento empresarial, sólo es posible en la medida en que se reconozcan las condiciones y circunstancias en que se configuran significados históricos de lo que se es y de lo que se quiere como organización.

La evaluación realizada sobre la historia de la formulación de la misión y la visión de la empresa, nos demostrará que las problemáticas hoy reconocidas

\section{Ricardo Ariza López:}

Sociólogo de la Universidad Santo Tomás con estudios en Psicología; Maestría en Planeación Socioeconómica; Especialización en Gerencia de Instituciones de Educación Superior; Maestría en Análisis de problemas económicos, políticos e internacionales contemporáneos. Exdecano de la Facultad de Sociología de la Universidad Santo Tomás, (2002-2004).

Exprofesor investigador y director del área en facultades de Comunicación Social en la Universidad Jorge Tadeo Lozano y Externado de Colombia. Director de trabajos de grado en comunicación y organización pública y privada.

Actualmente es profesor investigador del programa de Mercadeo y Publicidad de la Facultad de Ciencias de la Comunicación y Artes del Politécnico Grancolombiano, en Marketing holístico para la creación de plataformas estratégicas; y es profesor de la asignaturas Socioantropología del consumo, Nuevos lenguajes comunicacionales aplicados a publicidad y Publicidad, entre otras. Para contactar al autor: pangloss64@hotmail.com o ricardoarturo64@yahoo.com en los nuevos modelos de análisis empresarial desde la sistémica compleja, tienen sus raíces en la naturaleza "ideal" y "abstracta" de lo que la misión y la visión plantean. Esta idealización de lo comunicativo no ha permitido una autoorganización rápida de la empresa que le permita cerrarse como organización compleja adaptativa y abrirse informativamente a los diferentes mercados como una propuesta no sólo diferente, sino como un sistema de información que hace una diferencia; (es recoger el viejo principio de la complejidad de que una información es una diferencia que hace una diferencia. Las ventajas competitivas de una empresa no están en que se muestren como diferentes, sino en que hagan diferencias, y eso sólo es posible desde un concepto de sistema integral y holístico de su razón de ser y de su sentido de hacer).

El carácter "abstracto" e "ideal" del sentido de lo comunicativo de su visión y de su misión ha llevado a la necesidad de configurar otros mecanismos empresariales de carácter simbólico (creencias, valores y normas comunicativas) que tengan características más concretas y que son utilizadas cotidianamente para interpretar los diferentes sucesos y eventos del mercado. Este sistema de dispositivos simbólicos que surgen en el hacer cotidiano han ido dando forma a unas formas de pensar y actuar empresariales que son más concretas, específicas y efectivas.

Durante el proceso de evaluación se desea comprobar que la ausencia de sincronización sistémica de carácter complejo, entre el modelo ideal de comunicación inicial de carácter estructural que orienta la empresa y el territorio concreto de nuevos escenarios de mercado, es uno de los fenómenos que van a influir, en mayor grado, en las interdependencias de los diferentes componentes estratégicos, estructurales, culturales y de comportamiento empresarial derivados de la competencia en nuevos escenarios de mercado.

La ambigüedad de sentido comunicativo que genera la contradicción, entre lo abstracto y lo real, va creando también una idea conflictiva entre las direcciones estratégicas de la empresa que consiste en creer que el entorno los controla. Entonces no se logra comprender que el entorno no está diseñado para decirnos lo que debemos hacer; somos nosotros los que estamos diseñados para resolver lo que tenemos que hacer a partir 
del entorno indiferente. Esta situación va generando diferentes grados de conformismo entre las direcciones estratégicas de la empresa, tendiendo a limitar sus capacidades para buscar otros mecanismos de adaptación tanto internos como externos.

Las personas responsables de dar vida a nuevas formas de concebir la empresa como un sistema integral y holístico para solucionar la problemática de adaptación a nuevos mercados y lograr la supervivencia competitiva en los mismos, se apoyan en los conocimientos que surgen en las experiencias vividas. En sus prácticas cotidianas las personas que orientan las empresas han ido comprendiendo que:

La racionalidad implica dos tipos de cuestiones: la correspondiente a los fines y la específica de los medios. La racionalidad de medios sólo es un asunto de información empírica (de la clase de movimientos y medidas que llevan eficientemente -efficiently- a los objetivos). Pero la racionalidad de fines no es un asunto de información sino de legitimación. No está establecida únicamente por la indagación empírica, sino que incluye apreciación (apparaisal) y un juicio de valoración [la evaluación]. Y en el amplio esquema de las cosas, los dos aspectos se necesitan: los fines sin los medios adecuados son frustrantes; los medios sin fines adecuados improductivos e inútiles ${ }^{1}$.

\subsection{Los orígenes de los "conflictos comunicativos" de la empresa}

De acuerdo con la intencionalidad investigativa se podría intentar llevar a cabo un análisis con base en los principios comunicativos que expone el filósofo alemán Jürgen Habermas. Según Godet esta visión de la comunicación, a grandes rasgos, nos propone reconocer la importancia del "actuar comunicativo". Actuar comunicativo que permite a los hombres entenderse sobre valores y percepciones de mundo diferentes negociando definiciones comunes. Es conveniente recordar que alrededor del concepto de comunicación se despliegan: el patrón de autoorganización comunicativa (sentido); las finalidades; los objetivos; las estrategias; los resultados, las estructuras; la cultura; los comportamientos y los procesos de aprendizaje que dan forma a la empresa.
La comunicación, según la perspectiva teórica y epistemológica que sustenta este autor, vendría a ser un intento de ajuste que añade a la transmisión de información el juego de papeles y actos por los que los interlocutores se reconocen, actúan y fundan comunidades lingüísticas. Hablar es, en este caso, un intercambio de información y la realización de actos regidos por normas que pretenden transformar realidades.

Validación del sentido comunicativo de la empresa en todas sus dimensiones que debería tener en cuenta las circunstancias concretas del momento global y local de los nuevos mercados y la complejidad sistémica de la organización en la esfera de la competitividad para, en un intento de anticipación, prever qué es lo que realmente se puede lograr con las acciones comunicativas en la organización empresarial y así "determinar, teniendo en cuenta las fuerzas presentes, cuales podrían ser los valores del entorno, es decir, cuales son, independientemente de toda formalización, los escenarios posibles, realizables y deseables"2.

No se manifiestan respuestas formales y concretas a las preguntas: ¿Qué hacer para incidir? (acciones intencionales), ¿por qué razón hacerlo? (motivos de las acciones); ¿cómo hacerlo? (procesos); ¿con quién hacerlo? (responsables); ¿con qué hacerlo? (recursos).

La no existencia de respuestas concretas a las preguntas anteriormente mencionadas hace que los propósitos y las acciones de la visión y la misión vayan perdiendo sentido (dirección) e intención porque: "El problema consiste en saber si la relación entre la intención reflexionada y la acción es, a su vez, de naturaleza causal"3.

El estado de confusión generado por la abstracción, según la evaluación de la empresa "tradicionalmente concebida", se produce al: no identificar mundo exterior y realidad: el mundo exterior puede ser imaginado, en cuyo caso, junto con los lógicos se habla de mundo posible, lo cual no está muy lejos del universo de creencia en contraste con el mundo real. Este último engloba el "universo mental": se imponen de forma masiva a cualquier observador del mundo contemporáneo. Por todos lados oímos que nos encontramos en una civilización de la comunicación ${ }^{4}$.

1 Rescher, Nicolás. Razón y valores en la era científico-tecnológica. Barcelona: Paidos, Pág. 82.

2 Michel Godet. De la anticipación a la acción. México: Alfaomega, 1996.

3 Paul Ricoeur. El discurso de la acción. Madrid: Cátedra, 1981. Pág. 127. Se recomienda revisar todo el texto.

4 Christian Baylon y Xavier Mignot. La comunicación. Madrid: Cátedra, 1996. Pág. 67. Se recomienda la revisión de todo el texto. 
Una cosa es la empresa que se propone "hacer algo diferente" plasmado en sentidos de abstracción total y reforzada por estructuras administrativas, funcionales y operativas que no se modifican (que algunos analistas equiparan con una típica situación de "esquizofrenia organizacional"); y otra, una empresa que es coherente (incluidas sus contradicciones), sistémica, integral y holísticamente entre el pensar y el hacer, que se dan simultáneamente y son la misma cosa. ${ }^{5}$

Otros dos aspectos conflictivos están directamente vinculados con la autoorganización del sentido de identidad y el posicionamiento de la empresa. El primero de ellos se relaciona con el desconocimiento del concepto comunicativo que dirige las acciones, estructuras y estrategias constitutivas de los programas, proyectos y planes de la organización. El segundo tiene que ver con las dificultades de comprensión tanto del concepto como del sentido de comunicación que informa el sentido de la empresa. Las dificultades de comprensión tienen sus orígenes en el uso de un lenguaje comunicativo que invoca la visión y la misión, que propicia el tener que estar dando múltiples interpretaciones y explicaciones sobre lo que significa "mi empresa", "mi producto", "mi servicio". Es una empresa que tradicionalmente, ante la serie de ambigüedades "esquizoides" que delatan su situación de doble vínculo (odiar y amar al mismo tiempo a aquel de quien proviene la orientación de acción), necesita "explicar", de manera reiterada, que es una empresa diferente, "interesada en los nuevos retos y cambios que el mundo le impone", pero cuya actuación refleja las mismas incoherencias y limitaciones comprensivas de las organizaciones tradicionales. Se "comunica" un discurso, se "vive otra realidad", y se considera que ambas son compatibles. Cabría preguntarse aquí: si todo lo que hace una empresa dice de ella ¿cómo se resuelve tal situación de ambigüedad comunicativa ante las nuevas lógicas de competitividad en nuevos escenarios de mercado?

Por ejemplo, se puede determinar que la mayoría de las personas que hacen parte del enfoque de empresa tradicional, no han creado consciencia sobre cuál es el sentido comunicativo real y concreto que se supone que debería y debe orientar la finalidad, los objetivos, las estrategias, las estructuras, los resultados y las actividades de las que participan o a las que se encaminan. Por lo tanto, las personas dirigen sus actuaciones desde motivaciones, individuales o colectivas, que se derivan más de la experiencia o se sustentan en las capacidades profesionales individuales específicas y que reiteran la "profecía" del psicólogo de Palo Alto, Paul Watzlawick ${ }^{6}$, de que no hay nada más fácil para mantener una estructura organizativa que "hacer lo mismo de manera diferente", con el convencimiento para toda la organización de que realmente se está "innovando", cuando lo cierto es que se mantiene la misma dinámica estructural de acción sin haber modificado el pensar. Es lo que Jurgen Habermas ${ }^{7}$ llama una situación de comunicación sistemáticamente distorsionada:

* Las personas relacionadas con la empresa manifiestan que no comprenden el sentido de las nuevas interacciones que deben constituir, ni el sentido de lo que les dicen. En esta situación se hace problemática la comprensión de la comunicación y esto suscita la necesidad de estar interpretando constantemente lo que se dice o hace.

* Las personas cuestionan la verdad de lo que se les dice o lo que se hace. Este hecho problematiza la verdad de los actos comunicativos y ante esta situación, se tiene que estar dando constantes afirmaciones y explicaciones.

* Las personas no consideran como rectas las normas y su cumplimiento. En este caso se pone en duda la rectitud de los actos y esto conduce a tener que estar dando permanentes justificaciones.

* Las personas desconfían de la sinceridad en las actuaciones, y ponen en tela de juicio la veracidad de los actos. En esta situación se tiene que estar demostrando permanentemente la sinceridad de los actos.

Así se va creando una situación de comunicación sistemáticamente distorsionada que genera conflictos internos y externos que afectan el funcionamiento y la adaptación eficaz a los cambios del entorno y a la posibilidad de enfrentar competitivamente los nuevos escenarios de mercado.

\section{Procesos de aprendizaje comunicativos de la empresa}

El carácter comunicativo de la empresa significa que su finalidad, objetivo, estructura, resultados y actividades estén basadas en la elaboración conjunta y en el intercambio. Para lograr dicha elaboración e intercambio

5 En la vieja acepción del profesor Sebastián de Xavier Zubiri, sobre todo en su trabajo Estructura dinámica de la realidad. Madrid: Alianza Editorial, 1989.

6 Se recomienda revisar los libros: Cambio. Barcelona: Herder, 1993; ¿Es real la realidad? Barcelona: Herder, 1994; y El lenguaje del Cambio. Barcelona: Herder, 1994.

7 Se recomienda revisar de este autor: Teoría de la Acción Comunicativa I. Buenos Aires: Taurus, 1988; y Teoría de la Acción Comunicativa II. Buenos Aires: Taurus, 1987. 
cooperativo se deben propiciar las condiciones, los mecanismos y los dispositivos formales internos y externos que posibiliten un constante proceso de autoaprendizaje. Además de esto se debe destacar que los procesos formales de autoaprendizaje de la empresa facilitan el acceso a información útil del entorno interno y externo lo cual permite la adaptación al entorno porque hace flexible y dinámico su patrón de organización (sentido) y su configuración (estructura).

Los conocimientos, creencias, valores y prácticas que han demostrado ser efectivas para la interacción interna y externa están localizadas en la memoria colectiva histórica de las personas que hacen parte del todo de la empresa. Este saber hacer se socializa generalmente de manera informal y se aprende en los diálogos cotidianos diariamente entre ellos. En un modelo sistémico, integral y holístico de empresa siempre será pertinente que esta elaboración informal de los procesos de aprendizaje se estructure porque permite que los cambios esperados se den más rápidamente.

Establecido el nuevo sentido comunicativo concreto y realizable de la empresa, se debería dar forma a una estructura holística organizada. Esto debería incluir una reformulación general no solo de actividades, sino también y fundamentalmente, de objetivos, estrategias, y finalidades de la empresa de acuerdo con el sentido comunicativo escogido: la empresa que hace, dice de ella; todo lo que dice, es ella mientras lo hace. A eso llamamos: patrón de auto organización comunicativa.

Diseñar de acuerdo con el patrón de autoorganización y de la estructura holística de la empresa los mecanismos y dispositivos formales que permitan generar procesos de aprendizaje en la empresa, es la tarea comunicativa que se invoca en este tipo de propuesta investigativa. La primera acción que podría realizarse sería recuperar el saber hacer informal que le ha dado vida a la empresa. Saber hacer concreto que hasta ahora ha estado en la memoria individual de cada uno de sus integrantes y que debe socializarse a través de una sistematización de memoria formal y formalizante.

Varios autores e investigadores de la cibernética de segundo orden (Maturana y Espejo) ${ }^{8}$, postulan que las comunicaciones humanas se dan, en forma importante, en las organizaciones, en forma verbal, como conversaciones. "Rescatar" las conversaciones que se dan entre los diferentes actores de la empresa, debe hacerse en términos de memoria y de gestión, ya que las mismas combinan aspectos emocionales y afectivos, de manejo de poder, de líneas de autoridad, a los ya sugeridos arriba por los evaluadores de manejo de información y análisis de alternativas de decisión.

El investigador R. Espejo afirma, que estas conversaciones contribuyen a fortalecer los criterios de sentido de la organización y la apropiación de los mismos por parte de los interlocutores, haciendo referencia al entorno de manera dinámica y cambiante, y las compara con las necesidades y problemáticas internas de la organización.

Tener en cuenta los anteriores aspectos facilitaría a los diferentes actores de la empresa, el diseño de sistemas de control de gestión para reflexionar, sistemáticamente, sobre la comparación entre resultados y propósitos, y así cerrar más ágilmente sus ciclos de aprendizaje.

Los principios que orientarían estos ciclos de aprendizaje, comenzarían con la especificación del conjunto de requerimientos que le permitan a la empresa construir un modelo de sistema viable (MSV) tendiente a configurar una propuesta de autoorganización dinámica y adaptativa. El proceso de la formación sugerida se orienta por el algoritmo de Lander ${ }^{9}$ extensión:

1) Una solución conversacional al problema es un conjunto de propuestas validadas por el grupo, cada una de las cuales tiende a satisfacer uno o más requerimientos.

2) Cada solución o alternativa de solución del problema es iniciada por un negociador que conversa en alguno de los ciclos del proceso, y posteriormente es extendida y evaluada por los otros actores de la empresa durante ciclos futuros de procesamiento (memoria sistematizada). Por este motivo recibe el nombre de extensión incremental de soluciones.

3) A medida que se van generando propuestas, éstas van relacionándose entre sí para conformar soluciones. Al finalizar el proceso de resolución propiamente dicho, los actores de la empresa poseen un conjunto de

8 Beer, Stafford. Diagnosing the System for Organizations. Oxford: John Wiley \& Sons, 1985; Espejo, Raul. Strategy, Structure and Information Management. En: Journal of Information Systems, Vol. 3, No. 1, pp 17-31, 1991; Espejo, Raul. and R. Harnden (eds). The Viable System Model: Interpretations and Applications of Stafford Beer s VSM. Great Britain: John Wiley \& Sons Ltd, 1989; Maturana, Humberto. Biología del conocimiento, diferentes ediciones.

9 Lander,S. Distributed Search and Conflict Management Among Reusable Heterogeneous Agents, PhD Thesis, University of Massachusetts Amherst, May, 1994. 
soluciones o propuestas del cual deben seleccionar la o las que se orienten a dar respuesta al sentido de empresa como una organización estructurada de manera sistémica, integral y holística.

4) En una solución sólo hay una propuesta por requerimiento. Si en la negociación conversacional se han generado varias propuestas a un mismo requerimiento, cada una de ellas debe pertenecer a una solución diferente (sólo así se sostiene la dinámica de adaptación al contexto y al escenario socializante diferenciado).

5) Las soluciones no son necesariamente completas. Es posible que en una solución en particular queden requerimientos sin satisfacer.

6) Los negociadores conversacionales trabajan con y sobre argumentaciones para encadenar y sistematizar los requerimientos con las propuestas.

Al reconocer la gran importancia de las conversaciones en el patrón de autoorganización comunicativa de la empresa, se deben diferenciar entre las conversaciones del problema y las conversaciones de contexto de gestión. Las conversaciones del problema tratan directamente de la búsqueda de soluciones y se orientan por el tópico o asunto de la conversación ${ }^{10}$. Las conversaciones de contexto ${ }^{11}$ tratan sobre aspectos de la dinámica de negociación conversacional como actividad de trabajo y estructura.

Si la empresa inscribe su hilo conductor o transversal en los sentidos de identidad y posicionamiento organizativo (donde la información es una diferencia que hace una diferencia), deben observarse, de manera sistémica, los siguientes elementos de resolución en la negociación conversacional: negociador comunicativo, variable de decisión, requerimiento, propuesta.

Este dominio de la información permite el surgimiento de relaciones comunicativas que se traducen en los resultados y productos finales que la empresa disponga para esta acción con sentido de qué es lo que hace que dice la empresa: un patrón autoorganizativo comunicativo.

\section{Bibliografia}

- Baylon, Christian y Mignot, Xavier. La comunicación. Madrid, Cátedra, 1996.

- Beer, Stafford. Diagnosing the System for Organizations. Oxford, John Wiley \& Sons, 1985.

- Dijk,T.A.Von. Texto y Contexto. Madrid, Cátedra, 1980.

- Espejo, Raul. Strategy, Structure and Information Management. En: Journal of Information Systems, Vol. 3, No. 1, pp 17-31, 1991.

- Espejo, Raul y R. Harnden (eds). The Viable System Model: Interpretations and Applications of Stafford Beer s VSM. Great Britain, John Wiley \& Sons Ltd, 1989.

- Godet, Michel. De la anticipación a la acción. México, Alfaomega, 1996.

- Habermas, Jurgen. Teoría de la Acción Comunicativa I. Buenos Aires, Taurus, 1988.

- Habermas, Jurgen. Teoría de la Acción Comunicativa II. Buenos Aires, Taurus, 1987.

- Ingarden, R. Concreción y reconstrucción. En: Estética de la recepción de Rainer Warning (ed.) Madrid, Visor, 1989.

- Lander, S. Distributed Search and Conflict Management Among Reusable Heterogeneous Agents, PhD Thesis. University of Massachusetts, Amherst, May, 1994.

- Maturana, Humberto. Biología del conocimiento. En: Humberto Maturana y F. Varela. El árbol del conocimiento. Las bases biológicas del conocimiento humano. Madrid, Debate, 1980.

- Rescher, Nicolás. Razón y valores en la era científicotecnológica. Barcelona, Paidos, 2000.

- Ricoeur, Paul. El discurso de la acción. Madrid, Cátedra, 1981.

- Watzlawick, Paul. Cambio. Barcelona, Herder, 1993.

- Watzlawick, Paul. El lenguaje del Cambio. Barcelona, Herder, 1994.

- Watzlawick, Paul. ¿Es real la realidad? Barcelona, Herder, 1994. 